\title{
Neonatal outcome of suspected fetal distress cases
}

\author{
G Kaberi $^{1}$, A Fatema ${ }^{2}$
}

\begin{abstract}
Background: Perinatal mortality is closely related to the early and accurate recognition of foetal distress. Objective of the study was to find out neonatal outcome in suspected fetal distress cases. Methods: This was a cross sectional study done in ShSMCH. Purposively 38 samples were taken in three weeks time and clinical state related to fetal distress and neonatal outcome of them were observed. Results: Fetal movement were less in $36.8 \%$ cases. Abnormal fetal heart rate were present in $89.4 \%$ cases and in $47.4 \%$ cases amniotic fluid was meconium stained. First minute Apgar score was low in $36.8 \%$ cases and $47.4 \%$ were admitted in paediatric ward. The clinical profile for diagnosis of fetal distress was not significantly associated with low apgar scores. Conclusion: There was very little appreciation for assumption of fetal distress clinically. [J Shaheed Suhrawardy Med Coll 2016;8(1): 23-25]
\end{abstract}

Keywords: Fetal distress, Neonatal outcome

\section{Introduction}

Fetal distress is a widely used but poorly defined term. The diagnosis is usually based on characteristic changes in fetal heart rate assessed by auscultation or electronic recording with, in some cases, low $\mathrm{pH}$ in a fetal blood sample ${ }^{1-3}$. Suspected fetal distress during labour is always considered an emergency. The importance attached to it derives from the perceived association of fetal hypoxia (low oxygen levels) with perinatal morbidity/mortality and long term disability ${ }^{4}$. Intrapartum hypoxia complicates about $1 \%$ of labour and results in death in about 0.5 in 1000 pregnancies and cerebral palsy in 1 in 1000 pregnancies $^{5}$. The pathogenesis of intrapartum hypoxia is often multifactorial but poorly understood. Processes such as uteroplacental vascular disease, reduced uterine perfusion, fetal sepsis, reduced fetal reserves, and cord compression can be involved alone or in combination, and gestational and antepartum factors can modify the fetal response ${ }^{6}$. Methods of screening and diagnosing the condition have limitations ${ }^{7}$. When it is diagnosed clinically as "fetal distress" swift delivery is the aim, and the standard has become delivery within 30 minutes of diagnosing fetal distress ${ }^{8}$. In practice emergency caesarean section for fetal distress should be undertaken as quickly as possible and ideally within 30 minutes $^{9}$.

\section{Methodoogy}

Term pregnant women admitted in antenatal ward who fulfill the inclusion criteria were enrolled in the study. At the onset thorough history was taken and physical examination including obstetrical examinations were done. Gestational age was assessed by date of LMP, clinical examination and USG report at early weeks of pregnancy. Fetal distress was detected by history of fetal movement, detection of FHR \& rhythm, colour of liquor amni .Fetal movement was normal if it was more than 10 times in 12 hours period. Fetal heart rate was assumed to be normal if it was within 120-180 beats per minute and rhythm was taken as normal if the heart beats were in regular interval. If the fetal movement was less than 10 times in 12 hours period, intermittently auscultated fetal heart rate was more or less than normal, beats were irregular and amniotic fluid colour was meconium stained than the condition was diagnosed as fetal distress. Neonatal outcome was determined by Apgar score in 1 and 5 minute, birth weight $\&$ admission in pediatric ward.

1. Kaberi Guha, Junior Consultant, Obstetrics \& Gynaecology, Shaheed Suhrawardy Medical College Hospital, Sher-E-Bangla Nagar, Dhaka.

2. Fatema Asraf, Professor, Department of Obstetrics \& Gynaecology, Shaheed Suhrawardy Medical College Hospital, Sher-E-Bangla Nagar, Dhaka.

\section{Correspondence}

1.Dr. Kaberi Guha, Junior Consultant, Obstetrics \& Gynaecology, Shaheed Suhrawardy Medical College Hospital, Sher-E-Bangla Nagar, Dhaka. Mobile 01711802327,E mail guha.kaberi0@gmail.com 


\section{Results:}

Purposively 38 samples were taken in three weeks time. About 53\% cases maternal age group was within 15-19 years. Monthly expenditure revealed almost $42 \%$ were low socioeconomic group. Only $10.5 \%$ mother was service holder. Maternal perception of fetal movement were less in $36.8 \%$ cases. Abnormal fetal heart rate were present in $89.5 \%$ cases and in $47.4 \%$ cases amniotic fluid was meconium stained. First minute Apgar score was low in $36.8 \%$ cases and $47.4 \%$ were admitted in paediatric ward. The clinical profile for diagnosis of fetal distress was not significantly associated with low apgar scores.

Table1: Clinical State related to Fetal Distress $(n=38)$

\begin{tabular}{llcc}
\hline Clinical state related to fetal distress & Frequency & Percent \\
\hline Fetal & Normal & 24 & 63.2 \\
Movement & Less Fetal Movement & 14 & 36.8 \\
Fetal Heart & Normal & 4 & 10.5 \\
Rate & Abnormal & 34 & 89.5 \\
Fetal Heart & Regular & 32 & 84.2 \\
Rhythm & Irregular & 6 & 15.8 \\
Color of & Clear & 20 & 52.6 \\
Amniotic Fluid & Meconium Stained & 18 & 47.4 \\
\hline
\end{tabular}
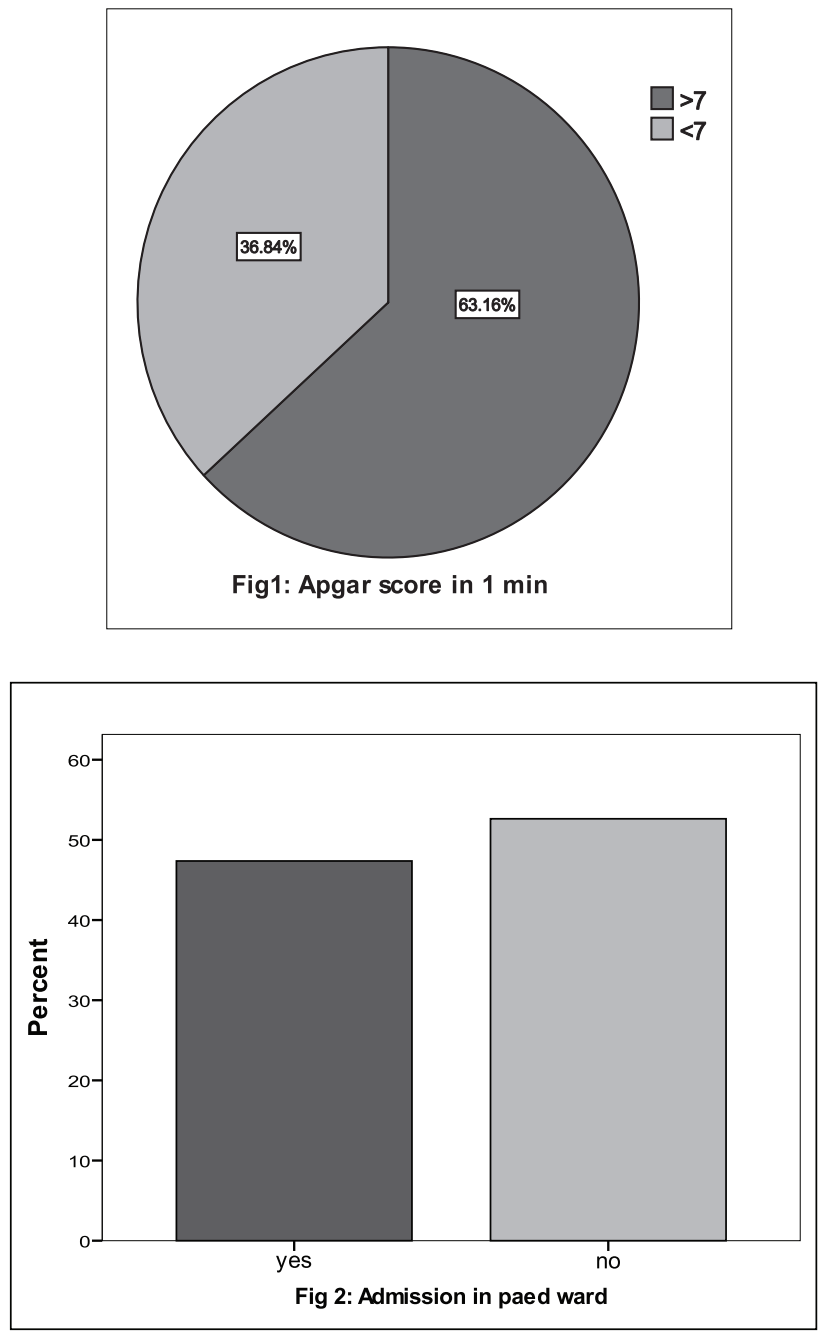

Table 2: Clinical profile of fetal distress compared with Apgar score in $1 \mathrm{~min}$

\begin{tabular}{llccc}
\hline Clinical profile of fetal distress & \multicolumn{3}{c}{ Apgar score in 1 } & p value \\
\cline { 3 - 4 } & & low & good & \\
\hline Fetal & Normal & 14 & 10 & $0.501 \mathrm{~ns}$ \\
Movement & Less than normal & 10 & 4 & \\
\multirow{2}{*}{ Fetal heart rate Normal } & 10 & 22 & $0.167 \mathrm{~ns}$ \\
& $\quad$ Abnormal & 4 & 2 & \\
\multirow{2}{*}{ Colour of } & Clear & & & \\
Amniotic Fluid & Meconium Stained & 6 & 18 & $0.253 \mathrm{~ns}$ \\
\hline
\end{tabular}

\section{Discussion}

For the present study only the clinical parameters were used for detecting fetal distress and also to assess neonatal outcome. We found that maternal history about fetal movement, fetal heart rate and rhythm and amniotic fluid colour were not significantly associated with low apgar scores. Another study also concluded that diagnosis of fetal heart rate was not significantly associated with low apgar scores so auscultation of fetal heart rate was an insensitive method of assessing the fetal condition and should not be used alone to diagnose fetal distress ${ }^{10}$.

In this study, in $52.6 \%$ cases colour of the amniotic fluid was clear. Miller FC et al found that although there was a 31/2 fold increase in incidence of low 5 min Apgar score in the meconium group but signs of fetal distress not significantly associated with colour of amniotic fluid ${ }^{11}$.

In many studies, both biochemical and clinical variables were used to assess the condition of newborn infants ${ }^{10}$. But in this set up only the Apgar scoring system is used. While Apgar scoring system is a popular method of assessing asphyxia, low scores relate poorly to fetal acidosis in labour ${ }^{12}$ and at delivery ${ }^{13}$ and to cerebral palsy in infancy ${ }^{14}$.

The $\mathrm{pH}$ is considered to be the most objective indication of fetal hypoxia during labour but the prognostic developmental implications of severe acidosis at birth have not been adequately evaluated ${ }^{10}$. A healthy fetus with an activated sympathetic nervous system may be able to respond successfully to a difficult labour, resulting in severe acidosis which is then rapidly reversed after delivery $^{15}$. On the other hand, a fetus compromised or with retarded growth before the onset of even a normal labour may not be able to adapt adequately ${ }^{16}$.

Deliveries by caesarean section might have been avoided on those occasions when there was not severe acidosis at birth had fetal blood sampling been used more often to assess the implication of the abnormal fetal heart rate patterns ${ }^{10}$.

In this study there was very little appreciation by clinicians for the dynamic nature of fetal adaptability to compromise and its reversibility. This study was done within a very short time and sample size was small. But this results may represent the obstetric practice of many hospitals in Bangladesh.

It is the responsibility of individual obstetric units to formulate their own policies as governed by their own 
resources and populations and to audit the value of their own obstetric management. This is particularly important in modern obstetrics, where induction and augmentations are common.

\section{References}

1. Goodlin RC. History of fetal monitoring. Am Jr Obstet Gynecol.1979; 133: 323-52

2. Zuspan FP, Quilligan EJ, James JD, van Geisn HP. Practices of intrapartum fetal obstetrics: the role of electronic fetal monitoring. Am J Obstet Gynecol.1979;135:287-91.

3. Beard RW, Filshie GM, Knight CA, Roberts GM. The significance of the changes of the continuous fetal heart rate in first stage labour. Journal of Obstetrics and Gynaecology of the British Commonwealth.1971;78:865-81. 4. Marlow N. The contribution of perinatal asphyxia in the term infant to outcomes in children. In: Roderick $\mathrm{CH}$, Whittle MJ editor(s). Fetal medicine-basic science and clinical practice. 4th Edition. London: Churchill Livingstone, 1999:1087-93.

5. Gaffney G, Sellers S, Flavell V, Squier M, Johnson A. A casecontrol study of intrapartum care, cerebral palsy, and perinatal death. BMJ1994;308:743505

6. Murphy D, Sellers S, MacKenzie IZ, Yudkin PL, Johnson A. A casecontrol study of antenatal and intrapartum risk factors for cerebral palsy in very preterm singleton babies. Lancet 1995;346:144954.

7. Thaker SB, Stroup DF. Continuous electronic heart rate monitoring for fetal assessment during labour. Cochrane Database of Systematic Reviews 2001;(1):CD000063.

8. David James.Caesarean section for fetal distress The 30 minute yardstick is in danger of becoming a rod for our backs Queen's Medical Centre, Nottingham NG7 2UH

9. National Institute for Clinical Excellence, Royal College of Obstetricians and Gynaecologists. The use of electronic fetal monitoring. London: RCOG, 2001.

10. Sykes GS, Molloy P, Johnson P, Stirrat GM, Turnbull AC. Fetal distress and the condition of newborn infants.BMJ.1983 Oct1;287: 943-45
11. Miller FC, Sacks DA, Yeh SY, Paul RH, Schifrin BS, Martin CB Jr, Hon EH. Significance of meconium during labor. Am J Obstet Gynecol. 1975 Jul 1;122(5):573-80.

12. Weber $\mathrm{T}$. The validity of discontinuous $\mathrm{pH}$ measurements on fetal blood and of cardiotocography in predicting neonatal Apgar scores. Dan Med Bull 1979;26:186-91.

13. Sykes GS, Molloy P, Johnson P, Gu W, Ashworth F, Stirrat GM, Turnbull AC. Do Apgar scores indicate asphyxia ? Lancet 1982;i :494-5.

14. Nelson KB, Ellenberg JH. Apgar scores as predictors of chronic neurologic disability. Pediatrics. $1981 ; 68: 36-44$.

15. Lagercrantz H. Asphyxia and the Apgar score. Lancet 1982;i:966.

16. Kenny JD, Garcia-Prats JA, Hilliard JL, Corbes JA, Rudolf JA. Hypercapnia at birth: a possible role in the pathogenesis of intra-ventricular hemorrhage. Pediatrics. 1978;62:465-7. levels of acute intoxication. J Formosan Med Assoc1984; 83:135-41.

31. Singh S, Sharma BK, Wahi PL, Anand BS, Chugh KS. Spectrum of acute poisonings in adults (10 year experience). J Assoc Physicians India1984; 32:561-3.

32. Gupta SK, Grover JK, Bhardwaj SL, Sahak T, Basu N. Blood barbiturate levels in 175 suspected suicide patients. J Assoc Physicians India1984; 32:340-2.

33. Yusuf HR, Akhter HH, Rahman MH, Chowdhury MK, Rochat RW. Injury-related deaths amongst women aged 10-50 years in Bangladesh, 1996-97. Lancet2000; 355:1220-4.

34. Ray JE, Reilly DK, Day RO. Drugs involved in self-poisoning: verification by toxicological analysis. Med J Aust. 1986 Apr 28; 144(9):455-7.

35. Malangu N. Characteristics of acute poisoning at two referral hospitals in Francistown and Gaborone. SA FamPract 2008; 50(3):67.

36. CharalambosTountas, AlexiosSotiropoulos, Stathis A Skliros, VasilikiKotsini, Theodoros A Peppas, Elias Tamvakos and Stavros Pappas. Voluntary self-poisoning as a cause of admission to a tertiary hospital internal medicine clinic in Piraeus, Greece within a year. BMC Psychiatry 2001; $1: 4$.

37. K.C. Yeung and T.W. Wong.Poisoning - An Epidemiological Study. Journal of the Hong Kong Medical Association1990; Vol. 42, No.4. 